\title{
Correlation between site preference and magnetic properties of Zn-Sn-substituted strontium hexaferrite
}

\author{
Vivek Dixit, ${ }^{1,2}$ Dinesh Thapa, ${ }^{1,2}$ Bipin Lamichhane, ${ }^{1,2}$ \\ Chandani N. Nandadasa, ${ }^{1,2}$ Yang-Ki Hong, ${ }^{3}$ and Seong-Gon Kim ${ }^{1,2, *}$ \\ ${ }^{1}$ Department of Physics and Astronomy, Mississippi State University, Mississippi State, MS 39762, USA \\ ${ }^{2}$ Center for Computational Sciences, Mississippi State University, Mississippi State, MS 39762, USA \\ ${ }^{3}$ Department of Electrical and Computer Engineering and MINT Center, \\ The University of Alabama, Tuscaloosa, AL 35487, USA
}

(Dated: November 21, 2018)

\begin{abstract}
The site preference and magnetic properties of $\mathrm{Zn}$, Sn and Zn-Sn substituted M-type strontium hexaferrite $\left(\mathrm{SrFe}_{12} \mathrm{O}_{19}\right)$ have been investigated using first-principles total energy calculations based on density functional theory. The site occupancy of substituted atoms were estimated by calculating the substitution energies of different configurations. The distribution of different configurations during the annealing process at high temperature was determined using the formation probabilities of configurations to calculate magnetic properties of substituted strontium hexaferrite. We found that the magnetization and magnetocrystalline anisotropy are closely related to the distributions of $\mathrm{Zn}-\mathrm{Sn}$ ions on the five Fe sites. Our calculation show that in $\mathrm{SrFe}_{11.5} \mathrm{Zn}_{0.5} \mathrm{O}_{19}$, Zn atoms prefer to occupy $4 f_{1}, 12 k$, and $2 a$ sites with occupation probability of $78 \%, 19 \%$ and $3 \%$, respectively, while in $\mathrm{SrFe}_{11.5} \mathrm{SnO}_{19}$, Sn atoms occupy the $12 k$ and $4 f_{2}$ sites with occupation probability of $54 \%$ and $46 \%$, respectively. We also found that in $\mathrm{SrFe}_{11} \mathrm{Zn}_{0.5} \mathrm{Sn}_{0.5} \mathrm{O}_{19},(\mathrm{Zn}, \mathrm{Sn})$ atom pairs prefer to occupy the $\left(4 f_{1}, 4 f_{2}\right),\left(4 f_{1}, 12 k\right)$ and $(12 k, 12 k)$ sites with occupation probability of $82 \%, 8 \%$ and $6 \%$, respectively. Our calculation shows that the increase of magnetization and the reduction of magnetic anisotropy in Zn-Sn substituted M-type strontium hexaferrite as observed experimentally is due to the occupation of $(\mathrm{Zn}, \mathrm{Sn})$ pairs at the $\left(4 f_{1}, 4 f_{2}\right)$ sites.
\end{abstract}

\section{INTRODUCTION}

Hexagonal strontium hexaferrite $\mathrm{SrFe}_{12} \mathrm{O}_{19}$ (SFO), along with other $M$-type hexaferrites $X_{\mathrm{Fe}_{12}} \mathrm{O}_{19}(X=$ $\mathrm{Sr}, \mathrm{Ba}, \mathrm{Pb})$, has large saturation magnetization, high coercivity, and excellent chemical stability [1-3]. Several experimental studies of substituted hexaferrite have been performed where magnetic properties are tailored to fit specific applications by the partial substitution of $\mathrm{Fe}$ in its crystallographic sites by divalent, trivalent, tetravalent, and divalent-tetravalent combination of metal atoms. The substitution of rare-earth elements such as $\operatorname{Pr}$ 4, 5, La 6, 7, Sm 8, and Nd 9] in M-type hexaferrite has shown to enhance the coercivity without much reduction in magnetization. Rare earth elements increase the spin-orbit coupling which in turn strengthen the magnetocrystalline anisotropy, hence the coercivity. However, reducing the use of rare-earth elements is highly desired for economical reasons. The substitution of $\mathrm{Al}$ leads to enhancement in coercivity 10 12. Wang et al. 13. have reported coercivity values as high as $17.6 \mathrm{kOe}$ for Al-substituted strontium hexaferrite. Even higher values of coercivity $(21.3 \mathrm{kOe})$ are possible by the double substitution of $\mathrm{Ca}$ and $\mathrm{Al}$ atoms [14, which is even higher than the coercivity of Nb-based magnets. The substitution of $\mathrm{Fe}$ by the trivalent metals such as $\mathrm{Al}$, $\mathrm{Ga}$, and $\mathrm{Cr}$ leads to an increase in coercivity and magnetocrystalline anisotropy (MAE) with reduction in magne-

\footnotetext{
* Corresponding author: sk162@msstate.edu
}

tization [12. In particular, the substitution of divalenttetravalent pairs such as Zn-Nb [15], Zn-Sn [16 19], and $\mathrm{Sn}-\mathrm{Mg}[2]$ result in significant enhancement in saturation magnetization with rapid reduction in coercivity.

Theoretical studies on pure and substituted M-type hexaferrite have also been performed. Fang et al. investigated the electronic structure of strontium hexaferrite using density-functional theory (DFT) 20]. Park et al. have calculated the exchange interaction of strontium hexaferrite from the differences of the total energy of different collinear spin configurations [21. Magnetism in La-substituted strontium hexaferrite has been studied using DFT [22, 23. Zn-Sn substituted strontium hexaferrite has been studied by Liyanage et al. 24]. The site occupancy and magnetic properties of $\mathrm{Al}, \mathrm{In}$, and Gasubstituted strontium hexaferrite has been investigated by Dixit et al. [10, 25].

In this work, we used first-principles total-energy calculations to investigate the relationship between the site occupation and magnetic properties of substituted strontium hexaferrite, $\mathrm{SrFe}_{12-x} M_{x} \mathrm{O}_{19}$ with $M=\mathrm{Zn}$ or $\mathrm{Sn}$ and $\mathrm{SrFe}_{12-2 x}(\mathrm{ZnSn})_{x} \mathrm{O}_{19}$ with $x=0.5$. The Boltzmann distribution function was used to determine the formation probabilities of various configurations at a typical annealing temperature (1000 K) of strontium hexaferrite, which was further used to compute the weighted average of various magnetic properties. We show that our model predicts an increase of saturation magnetization as well as a decrease in magnetic anisotropy energy (MAE) of $\mathrm{SrFe}_{11}(\mathrm{ZnSn})_{0.5} \mathrm{O}_{19}$ compared to the pure $M$-type SFO in good agreement with the experimental observations [19, 26]. 
(a)

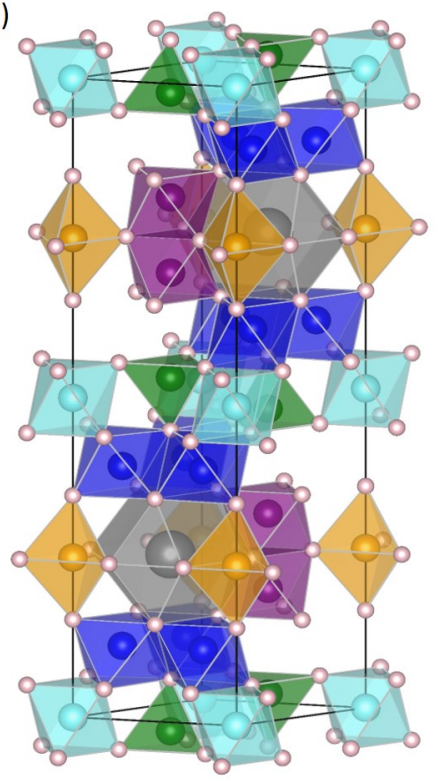

(b)



FIG. 1. (a) One double formula unit cell of $\mathrm{SrFe}_{12} \mathrm{O}_{19}$. Two large gray spheres are $\mathrm{Sr}$ atoms and small pink spheres are $\mathrm{O}$ atoms. Colored spheres enclosed by polyhedra formed by $\mathrm{O}$ atoms represent $\mathrm{Fe}^{3+}$ ions in different inequivalent sites: $2 a$ (cyan), $2 b$ (orange), $4 f_{1}$ (green), $4 f_{2}$ (purple), and $12 k$ (blue). (b) A schematic diagram of the lowest-energy spin configuration of $\mathrm{Fe}^{3+}$ ions of $\mathrm{SrFe}_{12} \mathrm{O}_{19}$. The arrows represent the local magnetic moment at each atomic site.

\section{METHODS}

SFO belongs to space group $P 6_{3} / m m c$ (No. 194) and has a hexagonal magnetoplumbite crystal structure 27. whose double formula unit cell containing 64 atoms is shown in Fig. 1. The unit cell structure of SFO consists of ten oxygen layers and the $\mathrm{Fe}^{+3}$ ions occupy five crystallographically inequivalent sites: three octahedral sites $\left(2 a, 12 k\right.$, and $\left.4 f_{2}\right)$, one tetrahedral site $\left(4 f_{1}\right)$, and one trigonal bipyramid site $(2 b)$ as indicated by the coordination polyhedra in Fig. 1(a). As a ferrimagnetic material, SFO has $16 \mathrm{Fe}^{+3}$ ions with spins in the majority direction $(2 a, 2 b$, and $12 k$ sites $)$ and $8 \mathrm{Fe}^{+3}$ ions with spins in the minority direction ( $4 f_{1}$ and $4 f_{2}$ sites) as shown in Fig. 1(b). First-principles total-energy calculations were performed to determine the site preference of $\mathrm{Zn}$ and $\mathrm{Sn}$ atoms in $M$-type Sr-hexaferrite. Total energies and forces were calculated using density-functional theory (DFT) with projector augmented wave (PAW) potentials as implemented in VASP [28, 29. The exchange correlation effect was described using the Perdew-BurkeErnzerhof (PBE) within generalized gradient approximation (GGA) [30. All calculations were spin polarized according to the ground state ferrimagnetic ordering of $\mathrm{Fe}$ spins [20, 31]. Electronic wave functions were expanded in a plane-wave basis with an energy cutoff of $520 \mathrm{eV}$. Reciprocal space was sampled with a $7 \times 7 \times 1$ Monkhorst-

Pack mesh [32] with a Fermi-level smearing of $0.2 \mathrm{eV}$ applied through the Methfessel-Paxton method [33] for relaxations and the tetrahedron method 34 for static calculations. Full geometrical optimization was performed to relax the positions of ions, cell shape, and cell volume until the largest force component on any ion was less than $0.01 \mathrm{eV} / \AA$. Since the on-site Coulomb interactions are particularly strong for localized $d$ electrons, we employed GGA+U method in the simplified rotationally invariant approach in order to avoid the self-interaction error in localized electron state of Fe-3d [35]. Based on our previous study [24, $U_{\text {eff }}$ for Fe atoms was set to $3.7 \mathrm{eV}$. The $U_{\text {eff }}$ for $\mathrm{Zn}\left(3 d^{10} 4 s^{2}\right)$, Sn $\left(5 s^{2} 5 p^{2}\right)$, Sr $\left(5 s^{2}\right)$, and $\mathrm{O}\left(2 s^{2} 2 p^{4}\right)$ were set to zero. Drawings in Fig. 1 are produced using VESTA code 36.

The magnetic properties of SFO can be modified by substitution of foreign atoms for Fe. There are five crystallographic inequivalent $\mathrm{Fe}$ sites in SFO. When foreign atoms are substituted in a SFO unit cell, there can be several energetically different configurations. Due to the ferrimagnetic nature of SFO, the magnetization of substituted SFO strongly depends on site preference of the substituted atoms. In order to investigate the effect of substitution on the magnetic properties, it is imperative to understand site preference of substituted atoms. The site preference of the substituted atom can be determined by calculating the substitution energy. The substitution energy $E_{\text {sub }}[i]$ for configuration $i$ at $0 \mathrm{~K}$ is given by

$$
E_{\mathrm{Sub}}[i]=E_{\mathrm{SFXO}}[i]-E_{\mathrm{SFO}}-\sum_{\alpha} n_{\alpha} \epsilon_{\alpha}
$$

where $E_{\mathrm{SFXO}}[i]$ is the total energy per unit cell of substituted SFO in configuration $i$, while $E_{\mathrm{SFO}}$ is the total energy per unit cell of pure SFO and $\epsilon_{\alpha}$ is the total energy per atom for element $\alpha(\alpha=\mathrm{Zn}$, Sn and $\mathrm{Fe})$ in its most stable crystal structure. Zn belongs to space group $P 6_{3} / m m c$ (No. 194) has a hexagonal crystal structure while Sn belongs to $F d \overline{3} m$ (No. 227) with cubic crystal system. $\epsilon_{Z n}, \epsilon_{S n}$ and $\epsilon_{F e}$ were found to be $-0.789 \mathrm{eV}$, $-3.835 \mathrm{eV}$ and $-8.461 \mathrm{eV}$, respectively. $n_{\alpha}$ is the number of atoms of type $\alpha$ added or removed; if one atom is added then $n_{\alpha}=+1$ while $n_{\alpha}=-1$ when one atom is removed.

The magnetocrystalline anisotropy energy, $E_{a}$, was also calculated. $E_{a}$ in the present case, is defined as the difference between the two total energies where the spin quantization axes are aligned along two different directions [37:

$$
E_{a}=E_{(100)}-E_{(001)}
$$

where, $E_{(100)}$ is the total energy with spin quantization axis in the magnetically hard plane and $E_{(001)}$ is the total energy with spin quantization axis in the magnetically easy axis. The total energies in Eq. (2) are computed by the non-self-consistent calculations, where the spin densities are kept constant 38. 
The uniaxial magnetic anisotropy constant, $K_{1}$, can be computed as [39, 40]:

$$
K_{1}=\frac{E_{a}}{V \sin ^{2} \theta}
$$

where $V$ is the equilibrium volume of the unit cell, and $\theta$ is the angle between the two spin quantization axis orientations $\left(90^{\circ}\right.$ in the present case). The anisotropy field, $H_{a}$, which is related to the coercivity can be expressed as [41]:

$$
H_{a}=\frac{2 K_{1}}{M_{s}}
$$

where $K_{1}$ is the magnetocrystalline anisotropy constant and $M_{s}$ is the saturation magnetization.

When the separation between $E_{\text {sub }}$ of different configurations is not too big compared to the thermal energy during the synthesis of these hexaferrites at a high annealing temperature $(\gtrsim 1000 \mathrm{~K})$, we can expect the site preference of substituted atoms to change at such an elevated temperature. This change in the site occupation preference can be modeled using Maxwell-Boltzmann distribution. The site occupation probability or the formation probability $P_{i}(T)$ of configuration $i$ at temperature $T$ is given by

$$
\begin{gathered}
P_{i}(T)=\frac{g_{i} \exp \left(-\Delta G_{i} / k_{B} T\right)}{\sum_{j} g_{j} \exp \left(-\Delta G_{j} / k_{B} T\right)} \\
\Delta G_{i}=\Delta E_{\mathrm{i}}+P \Delta V_{i}-T \Delta S_{i} \\
\Delta S_{i}=k_{B} \ln \left(g_{i}\right)-k_{B} \ln \left(g_{0}\right)
\end{gathered}
$$

where $\Delta G_{i}, \Delta E_{\mathrm{i}}, \Delta V_{i}$, and $\Delta S_{i}$ are the change in free energy, substitution energy, unit cell volume and entropy of the configuration $i$ relative to the ground state configuration. $P, k_{\mathrm{B}}$ and $g_{i}$ are the pressure, Boltzmann constant and multiplicity of configuration $i . g_{0}$ is the multiplicity of the ground state configuration. In our earlier work, we considered $\Delta S_{i}$ to be the same for all configurations [42]. Eq. (7) improves the model by explicit calculation of change in entropy with respect to the most stable configuration 43 .

Therefore, when the formation probability of higher energy configurations become non-negligible at the annealing temperature, it can be concluded that in a sample of substituted SFO there will not be a single configuration but a distribution of several configurations. Any physical quantity of a sample of SFO will then be a weighted average of respective quantity in different configurations:

$$
\langle Q\rangle=\sum_{i} P_{1000 \mathrm{~K}}(i) \cdot Q_{i}
$$

where $P_{1000 \mathrm{~K}}(i)$ and $Q_{i}$ are the formation probability at $1000 \mathrm{~K}$ and a physical quantity $Q$ of the configuration $i$. During the annealing process when the material is maintained at high temperature, substituted atoms have sufficient energy to overcome the local energy barriers and acquire energetically the various configurations that have sufficient formation probability. We note that the substituted SFO considered in the present work loses its magnetic properties at a typical annealing temperature (1000 K or higher) that is near or above its Curie temperature. When the material is cooled down to a low temperature below the critical temperature, it regains the magnetic properties while the configurations are locked in those with higher substitution energy due to energy barriers between them. Consequently, the weighted average calculated by Eq. (8) is the material's low temperature property even though $P_{1000 \mathrm{~K}}$ is used for computation.

\section{RESULTS AND DISCUSSION}

In this work, two types of substitutions have been studied. In the first case one $\mathrm{Zn}$ or Sn atom was substituted in a SFO unit cell. In the second case, a pair of $\mathrm{Zn}$ and Sn atoms were substituted in a SFO unit cell. Although there are $24 \mathrm{Fe}$ atoms in SFO unit cell, the application of crystallographic symmetry operations shows that many of these Fe sites are equivalent and leaves only five inequivalent sites. Therefore, one foreign atom can be substituted in five different ways, giving rise to five different configurations. We label these inequivalent configurations using the crystallographic name of the Fe site: $[2 a]$, $[2 b],\left[4 f_{1}\right],\left[4 f_{2}\right]$, and $[12 k]$. $E_{\mathrm{sub}}$ corresponding to the substitution of one $\mathrm{Zn}$ at different inequivalent $\mathrm{Fe}$ sites is given in Table I. The lowest $E_{\text {sub }}$ was found when a Zn atom was substituted at $4 f_{1}$ site, followed by the $[2 a]$ and $[12 k]$ cases. For the case of a single Sn atom substitution (Table II the lowest $E_{\text {sub }}$ was found to be for the substitution at the $4 f_{2}$ site, and the second lowest $E_{\text {sub }}$ was corresponding to the substitution at the $12 k$ site. A high multiplicity of the $12 k$ site and low $E_{\text {sub }}$ indicate that at higher temperatures the $12 k$ site is very likely to be occupied. In the second case, where a pair of Zn and Sn atoms were substituted in the SFO unit cell, there can be $5 \times 5=25$ different configurations. $E_{\text {sub }}$ corresponding to all these configurations are listed in Table III The lowest $E_{\text {sub }}$ was found to be the configuration where $\mathrm{Zn}$ goes to the $4 f_{1}$ while $\mathrm{Sn}$ occupies the $4 f_{2}$ site.

Fig. 2 shows the variation of site occupation probability with temperature for the inequivalent configurations of $\mathrm{SrFe}_{12-x} \mathrm{Zn}_{x} \mathrm{O}_{19}$ with $x=0.5$. In low temperature range the $4 f_{1}$ site is most likely to be occupied. However, as temperature rises this probability falls while the site occupation probability of $12 k$ rises. This is due to small substitution energy difference between $4 f_{1}$ and $12 k$ sites and higher multiplicity of the $12 k$ site. Similar behavior of site occupancy was seen in the case of single Sn atom substitution as shown in Fig. 3. In the elevated temperature regime, the site occupancy of the $4 f_{2}$ falls, while that of the $12 k$ site rises. In fact, at the annealing temperature of SFO (1000 K), the occupation prob- 
TABLE I. Physical properties of inequivalent configurations of $\mathrm{SrFe}_{12-x} \mathrm{Zn}_{x} \mathrm{O}_{19}$ with $x=0.5$ : multiplicity ( $g$ ), substitution energy $\left(E_{\mathrm{sub}}\right)$, volume of the unit cell $(V)$, total magnetic moment $\left(m_{\text {tot }}\right)$, saturation magnetization $\left(\sigma_{s}\right)$, magnetocrystalline anisotropy energy $\left(E_{a}\right)$, uniaxial magnetic anisotropy constant $\left(K_{1}\right)$, anisotropy field $\left(H_{a}\right)$, and the formation probability at $1000 \mathrm{~K}\left(P_{1000 \mathrm{~K}}\right)$. All values are for a double formula unit cell containing 64 atoms.

\begin{tabular}{cccccccccc}
\hline \hline config & $g$ & $E_{\text {sub }}(\mathrm{eV})$ & $V\left(\AA^{3}\right)$ & $m_{\text {tot }}\left(\mu_{\mathrm{B}}\right)$ & $\sigma_{s}(\mathrm{emu} / \mathrm{g})$ & $E_{a}(\mathrm{meV})$ & $K_{1}\left(\mathrm{~kJ} / \mathrm{m}^{3}\right)$ & $H_{a}(\mathrm{kOe})$ & $P_{1000 \mathrm{~K}}$ \\
\hline$\left[4 f_{1}\right]$ & 4 & -1.44 & 706.61 & 44 & 115.01 & 0.83 & 188.20 & 6.37 \\
{$[2 a]$} & 2 & -1.27 & 705.15 & 34 & 88.74 & 0.90 & 204.49 & 8.95 \\
{$[12 k]$} & 12 & -1.13 & 706.61 & 34 & 88.70 & 0.80 & 181.39 & 0.025 \\
{$[2 b]$} & 2 & -0.86 & 708.08 & 36 & 94.13 & 0.63 & 142.55 & 5.96 \\
{$\left[4 f_{2}\right]$} & 4 & -0.63 & 708.14 & 44 & 115.15 & 0.85 & 192.31 & 6.52 & 0.000 \\
\hline \hline
\end{tabular}

TABLE II. Physical properties of inequivalent configurations of $\mathrm{SrFe}_{12-x} \mathrm{Sn}_{x} \mathrm{O}_{19}$ with $x=0.5$ : multiplicity ( $g$ ), substitution energy $\left(E_{\text {sub }}\right)$, volume of the unit cell $(V)$, total magnetic moment $\left(m_{\text {tot }}\right)$, saturation magnetization $\left(\sigma_{s}\right)$, magnetocrystalline anisotropy energy $\left(E_{a}\right)$, uniaxial magnetic anisotropy constant $\left(K_{1}\right)$, anisotropy field $\left(H_{a}\right)$, and the formation probability at $1000 \mathrm{~K}\left(P_{1000 \mathrm{~K}}\right)$. All values are for a double formula unit cell containing 64 atoms.

\begin{tabular}{cccccccccc}
\hline \hline config & $g$ & $E_{\text {sub }}(\mathrm{eV})$ & $V\left(\AA^{3}\right)$ & $m_{\text {tot }}\left(\mu_{\mathrm{B}}\right)$ & $\sigma_{s}(\mathrm{emu} / \mathrm{g})$ & $E_{a}(\mathrm{meV})$ & $K_{1}\left(\mathrm{~kJ} / \mathrm{m}^{3}\right)$ & $H_{a}(\mathrm{kOe})$ & $P_{1000 \mathrm{~K}}$ \\
\hline$\left[4 f_{2}\right]$ & 4 & -2.42 & 716.94 & 44 & 112.37 & 0.66 & 147.49 & 5.06 \\
{$[12 k]$} & 12 & -2.25 & 716.65 & 36 & 91.95 & 0.83 & 185.56 & 7.77 \\
{$[2 b]$} & 2 & -1.78 & 712.67 & 34 & 86.74 & 0.66 & 148.38 & 6.538 \\
{$\left[4 f_{1}\right]$} & 4 & -1.51 & 715.41 & 44 & 112.38 & 0.18 & 40.31 & 1.38 & 0.000 \\
{$[2 a]$} & 2 & -1.51 & 716.45 & 35 & 90.65 & 0.90 & 201.27 & 8.55 & 0.000 \\
\hline \hline
\end{tabular}

TABLE III. Physical properties of inequivalent configurations of $\mathrm{SrFe}_{12-2 x}(\mathrm{ZnSn})_{x} \mathrm{O}_{19}$ with $x=0.5$ : multiplicity $(g)$, substitution energy $\left(E_{\mathrm{sub}}\right)$, volume of the unit cell $(V)$, total magnetic moment $\left(m_{\text {tot }}\right)$, saturation magnetization $\left(\sigma_{s}\right)$, magnetocrystalline anisotropy energy $\left(E_{a}\right)$, uniaxial magnetic anisotropy constant $\left(K_{1}\right)$, anisotropy field $\left(H_{a}\right)$, and the formation probability at $1000 \mathrm{~K}\left(P_{1000 \mathrm{~K}}\right)$. All values are for a double formula unit cell containing 64 atoms.

\begin{tabular}{|c|c|c|c|c|c|c|c|c|c|}
\hline config & $g$ & $E_{\text {sub }}(\mathrm{eV})$ & $V\left(\AA^{3}\right)$ & $m_{\text {tot }}\left(\mu_{\mathrm{B}}\right)$ & $\sigma_{s}(\mathrm{emu} / \mathrm{g})$ & $E_{a}(\mathrm{meV})$ & $K_{1}\left(\mathrm{~kJ} / \mathrm{m}^{3}\right)$ & $H_{a}(\mathrm{kOe})$ & $P_{1000 \mathrm{~K}}$ \\
\hline$\left[4 f_{1}, 4 f_{2}\right]$ & 16 & -5.326 & 717.32 & 50 & 127.13 & 0.70 & 156.35 & 4.72 & 0.819 \\
\hline$\left[2 b, 4 f_{2}\right]$ & 8 & -5.124 & 719.68 & 40 & 101.97 & 0.58 & 129.12 & 4.88 & 0.020 \\
\hline$\left[4 f_{1}, 2 a\right]$ & 8 & -5.065 & 716.49 & 40 & 101.68 & 0.82 & 183.36 & 6.91 & 0.010 \\
\hline$\left[2 a, 4 f_{2}\right]$ & 8 & -4.991 & 714.67 & 40 & 101.72 & 0.82 & 183.83 & 6.91 & 0.004 \\
\hline$\left[4 f_{1}, 12 k\right]$ & 48 & -4.940 & 717.54 & 40 & 101.72 & 0.61 & 136.21 & 5.14 & 0.084 \\
\hline$[12 k, 12 k]$ & 132 & -4.736 & 715.33 & 30 & 76.17 & 0.77 & 172.46 & 8.67 & 0.060 \\
\hline$[2 a, 12 k]$ & 24 & -4.697 & 715.08 & 30 & 76.28 & 0.83 & 185.97 & 9.33 & 0.001 \\
\hline$\left[12 k, 4 f_{2}\right]$ & 48 & -4.663 & 717.04 & 40 & 101.58 & 0.63 & 140.77 & 5.32 & 0.003 \\
\hline$\left[4 f_{1}, 2 b\right]$ & 8 & -4.650 & 715.16 & 40 & 101.72 & 0.49 & 109.78 & 4.13 & 0.000 \\
\hline$[12 k, 2 a]$ & 24 & -4.366 & 718.08 & 30 & 76.17 & 0.71 & 158.42 & 7.99 & 0.000 \\
\hline$[12 k, 2 b]$ & 24 & -4.207 & 715.13 & 30 & 76.15 & 0.49 & 109.78 & 5.52 & 0.000 \\
\hline$\left[2 a, 4 f_{1}\right]$ & 8 & -4.206 & 714.79 & 40 & 101.68 & 0.90 & 210.73 & 7.59 & 0.000 \\
\hline$[2 a, 2 a]$ & 2 & -4.156 & 720.35 & 30 & 76.26 & 0.85 & 189.05 & 9.56 & 0.000 \\
\hline$[2 b, 12 k]$ & 24 & -4.119 & 721.64 & 30 & 76.39 & 0.58 & 128.77 & 6.51 & 0.000 \\
\hline$\left[4 f_{2}, 2 b\right]$ & 8 & -4.082 & 716.07 & 40 & 101.73 & 0.53 & 118.59 & 4.47 & 0.000 \\
\hline$\left[4 f_{1}, 4 f_{1}\right]$ & 12 & -3.946 & 720.60 & 50 & 127.17 & 0.78 & 173.43 & 5.26 & 0.000 \\
\hline$\left[12 k, 4 f_{1}\right]$ & 48 & -3.932 & 717.21 & 40 & 101.59 & 0.84 & 187.65 & 7.09 & 0.000 \\
\hline$\left[4 f_{2}, 4 f_{2}\right]$ & 12 & -3.876 & 721.34 & 50 & 127.18 & 0.72 & 159.92 & 4.85 & 0.000 \\
\hline$[2 a, 2 b]$ & 4 & -3.726 & 717.56 & 30 & 76.58 & 0.79 & 176.39 & 8.83 & 0.000 \\
\hline$[2 b, 2 a]$ & 4 & -3.706 & 717.50 & 30 & 76.58 & 0.79 & 175.79 & 8.85 & 0.000 \\
\hline$\left[4 f_{2}, 12 k\right]$ & 48 & -3.586 & 723.09 & 40 & 101.86 & 0.75 & 166.12 & 6.31 & 0.000 \\
\hline$\left[4 f_{2}, 2 a\right]$ & 8 & -3.493 & 724.60 & 40 & 101.73 & 1.12 & 246.98 & 9.41 & 0.000 \\
\hline$\left[2 b, 4 f_{1}\right]$ & 8 & -3.297 & 722.28 & 40 & 101.83 & 0.68 & 150.84 & 5.73 & 0.000 \\
\hline$[2 b, 2 b]$ & 2 & -3.086 & 690.64 & 30 & 76.36 & 0.41 & 95.11 & 4.60 & 0.000 \\
\hline$\left[4 f_{2}, 4 f_{1}\right]$ & 16 & -3.073 & 723.67 & 50 & 127.21 & 0.78 & 172.69 & 5.26 & 0.000 \\
\hline
\end{tabular}




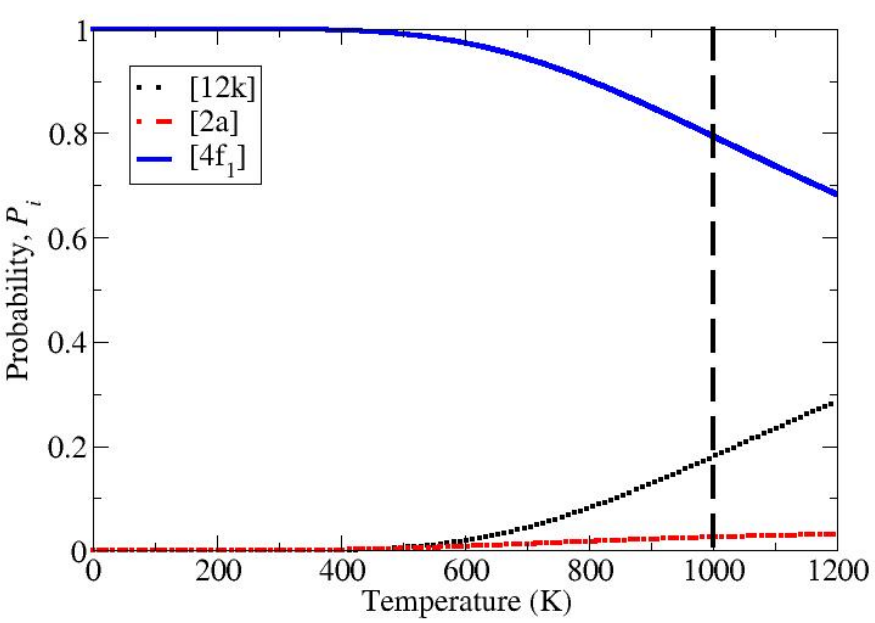

FIG. 2. Temperature dependence of the formation probability of different configurations of $\mathrm{SrFe}_{11.5} \mathrm{Zn}_{0.5} \mathrm{O}_{19}$. The configurations with negligible probability are not shown. The vertical dotted line indicates the annealing temperature of $1000 \mathrm{~K}$.

ability of the $12 k$ site $(54 \%)$ becomes higher than that of the $4 f_{2}$ site $(46 \%)$, which is the most energetically stable substitution site at $0 \mathrm{~K}$. For the Zn-Sn substitution $\left(\mathrm{SrFe}_{11} \mathrm{Zn}_{0.5} \mathrm{Sn}_{0.5} \mathrm{O}_{19}\right)$, the formation probability of $\left[4 f_{1}, 4 f_{2}\right]$ is high in the low as well as high temperature range. Other important configurations with significant formation probability at high temperature $(1000 \mathrm{~K})$ are $\left[4 f_{1}, 12 k\right]$ and $[12 k, 12 k]$ (Fig. 44. Substituted Zn atoms are most likely to occupy $4 f_{1}$ and $12 k$ sites, while Sn atoms occupy $4 f_{2}$ and $12 k$ sites.

The total magnetic moment $m_{\text {tot }}$ upon the substitution of a single $\mathrm{Zn}$ and $\mathrm{Sn}$ atom at various $\mathrm{Fe}$ site of $\mathrm{SFO}$ is given in Table [ and Table II It is evident that the substitution at the minority spin sites $\left(4 f_{1}\right.$ and $\left.4 f_{2}\right)$ enhances the net magnetic moment, while moment is reduced for the substitution at the majority spin sites $(12 k, 2 a$, and $2 b)$. Similar changes in the total magnetic moment were also noticed for $\left(\mathrm{SrFe}_{11} \mathrm{Zn}_{0} .5 \mathrm{Sn}_{0.5} \mathrm{O}_{19}\right.$ substitution (Table III). The saturation magnetization $\sigma_{s}$ values are also listed.

$E_{a}, K_{1}$, and $H_{a}$ of various configurations are presented in Table [1] III and III. In these cases, substitutions at $2 a$ site seems to enhance the anisotropy values. Table III also that the reduction of magnetic coercivity in $\mathrm{SrFe}_{11}(\mathrm{ZnSn})_{0.5} \mathrm{O}_{19}$ as experimentally observed [16 is mainly due to the occupation of $(\mathrm{Zn}, \mathrm{Sn})$ pair at the $\left(4 f_{1}, 4 f_{2}\right)$ sites. Although the $\left[2 b, 4 f_{2}\right]$ configuration, the one with second lowest substitution energy at $0 \mathrm{~K}$, has much lower $K_{1}$ and $H_{a}$ values, its multiplicity is much lower compared to other low-energy configurations and its formation probability grows merely to $2 \%$ (compared to $82 \%$ for the $\left[4 f_{1}, 4 f_{2}\right]$ configuration) even at $1000 \mathrm{~K}$.

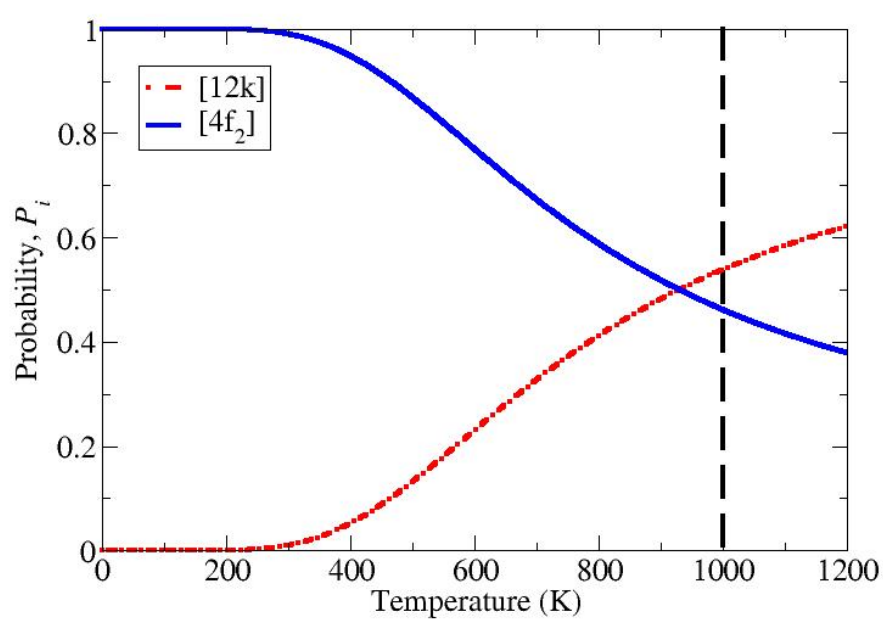

FIG. 3. Temperature dependence of the formation probability of different configurations of $\mathrm{SrFe}_{11.5} \mathrm{Sn}_{0.5} \mathrm{O}_{19}$. The configurations with negligible probability are not shown. The vertical dotted line indicates the annealing temperature of $1000 \mathrm{~K}$.

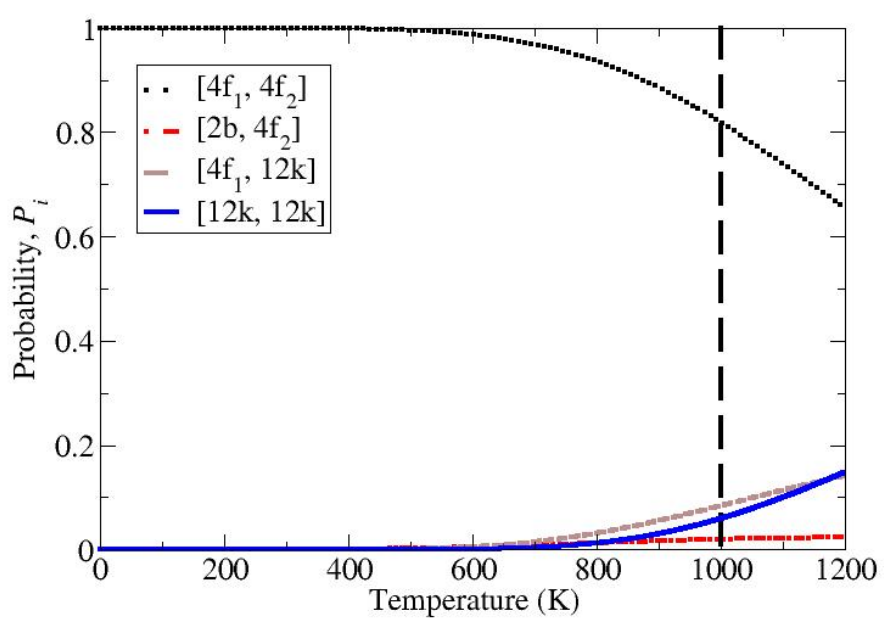

FIG. 4. Temperature dependence of the formation probability of different configurations of $\mathrm{SrFe}_{11.0} \mathrm{Zn}_{0.5} \mathrm{Sn}_{0.5} \mathrm{O}_{19}$. The configurations with negligible probability are not shown. The vertical dotted line indicates the annealing temperature of $1000 \mathrm{~K}$.

This is a different behavior from the related systems $\mathrm{BaFe}_{12-x}\left(\mathrm{Zr}_{0.5} \mathrm{Zn}_{0.5}\right)_{x} \mathrm{O}_{19}$ and $\mathrm{LaZnFe}_{11} \mathrm{O}_{19}$, where the $2 b$ site plays the main role in the reduction of anisotropy [44, 45.

Table IV shows the weighted average of physical properties of pure and substituted (Zn, Sn, Zn-Sn) strontium hexaferrite based on the formation probabilities 
TABLE IV. Weighted averages of physical properties of pure and substituted (Zn, Sn, Zn-Sn) strontium hexaferrite: volume of the unit cell $(V)$, total magnetic moment $\left(m_{\text {tot }}\right)$, saturation magnetization $\left(\sigma_{s}\right)$, magnetocrystalline anisotropy energy $\left(E_{a}\right)$, uniaxial magnetic anisotropy constant $\left(K_{1}\right)$, and anisotropy field $\left(H_{a}\right)$.

\begin{tabular}{ccccccc}
\hline \hline material & $\langle V\rangle\left(\AA^{3}\right)$ & $\left\langle m_{\text {tot }}\right\rangle\left(\mu_{\mathrm{B}}\right)$ & $\left\langle\sigma_{s}\right\rangle(\mathrm{emu} / \mathrm{g})$ & $E_{a}(\mathrm{meV})$ & $K_{1}\left(\mathrm{~kJ} / \mathrm{m}^{3}\right)$ & $H_{a}(\mathrm{kOe})$ \\
\hline $\mathrm{SrFe}_{12} \mathrm{O}_{19}$ & 706.83 & 39.0 & 110.19 & 0.85 & 193.00 & 7.35 \\
$\mathrm{SrFe}_{11.5} \mathrm{Zn}_{0.5} \mathrm{O}_{19}$ & 706.83 & 41.7 & 109.22 & 0.83 & 187.39 & 6.75 \\
$\mathrm{SrFe}_{11.5} \mathrm{Sn}_{0.5} \mathrm{O}_{19}$ & 718.61 & 39.7 & 101.34 & 0.75 & 168.05 & 6.52 \\
$\mathrm{SrFe}_{11}(\mathrm{ZnSn})_{0.5} \mathrm{O}_{19}$ & 717.08 & 47.2 & 120.01 & 0.69 & 153.87 & 4.99 \\
\hline \hline
\end{tabular}

TABLE V. Comparison of calculated and experimental magnetic properties of $\operatorname{SrFe}_{12-2 x}(\mathrm{ZnSn})_{x} \mathrm{O}_{19}$ with $x=0$ and $x=0.5$ : saturation magnetization $\left(\sigma_{s}\right)$, uniaxial magnetic anisotropy constant $\left(K_{1}\right)$, and anisotropy field $\left(H_{a}\right)$. The calculated values are for $0 \mathrm{~K}$ while the experimental values are measured at the room temperature. Relative difference w.r.t. pure SFO $(x=0)$ values are given in parentheses.

\begin{tabular}{|c|c|c|c|c|c|c|}
\hline & \multicolumn{2}{|c|}{$\sigma_{s}(\mathrm{emu} / \mathrm{g})$} & \multicolumn{2}{|c|}{$K_{1}\left(\mathrm{~kJ} / \mathrm{m}^{3}\right)$} & \multicolumn{2}{|c|}{$H_{a}(\mathrm{kOe})$} \\
\hline & $x=0$ & $x=0.5$ & $x=0$ & $x=0.5$ & $x=0$ & $x=0.5$ \\
\hline Exp. [16, 18 & 69.9 & $75.1(+7.4 \%)$ & 280.9 & $210.7(-25.0 \%)$ & 18.7 & $12.5(-33.0 \%)$ \\
\hline Calc. [This work] & 110.2 & $120.0(+8.2 \%)$ & 193.0 & $153.9(-20.0 \%)$ & 7.4 & $5.0(-32.0 \%)$ \\
\hline Calc. 24 & 113.1 & $127.2(+12.5 \%)$ & 190.0 & $100.0(-47.4 \%)$ & 7.1 & $3.0(-57.0 \%)$ \\
\hline
\end{tabular}

at the annealing temperature. In all three cases of substituted SFO, the $m_{\text {tot }}$ was estimated to be greater than that of pure SFO. Biggest increase was found in the case of Zn-Sn pair substituted SFO. A reduction in the values of $\sigma_{s}$ and anisotropy of $\mathrm{SrFe}_{11.5} \mathrm{Zn}_{0.5} \mathrm{O}_{19}$ and $\mathrm{SrFe}_{11.5} \mathrm{Sn}_{0.5} \mathrm{O}_{19}$ is in agreement with previous experimental studies on substituted barium hexaferrite [46, 47]. The saturation magnetization $\left(\sigma_{s}\right)$ of $\mathrm{SrFe}_{11.5} \mathrm{Zn}_{0.5} \mathrm{O}_{19}$ and $\mathrm{SrFe}_{11} \mathrm{Zn}_{0.5} \mathrm{Sn}_{0.5} \mathrm{O}_{19}$ were higher than that of pure SFO. In Table V we compare the magnetic properties of Zn-Sn pair substituted SFO in this work with previously reported computed and experimental data. Results from the present work show an increase of $8.2 \%$ in the value of $\sigma_{s}$, while Ghasemi et al. 18] experimentally observed a similar increase of $7.4 \%$. Quantities related to magnetic anisotropy viz. $K_{1}$, and $H_{a}$ showed reduction compared to pure $\mathrm{SFO}$. $\mathrm{SrFe}_{11} \mathrm{Zn}_{0.5} \mathrm{Sn}_{0.5} \mathrm{O}_{19}$ showed a reduction of $20 \%$ and $32 \%$ in $K_{1}$ and $H_{a}$, respectively, while experimentally Fang et al. observed a reduction of $25 \%$ and $33 \%$ in $K_{1}$ and $H_{a}$ values [16]. Table V]indicates that our present results are in better agreement with experimental results compared to the previous computed results that was obtained by considering the ground state configurations at $0 \mathrm{~K}$ only. Thus, our model based on the formation probability at the annealing temperature successfully explains the reduction in anisotropy of $\mathrm{Zn}-\mathrm{Sn}$ substituted SFO as well as the increase in magnetization values.

\section{CONCLUSION}

Using first-principles total energy calculations based on density functional theory, we calculated substitution energies of Zn, Sn, and Zn-Sn pair substituted strontium hexaferrite. These energy values were used to determine site preferences of the substituted atoms at $0 \mathrm{~K}$ as well as at a high annealing temperature. The site occupation probabilities or the formation probabilities of different configurations were then used to estimate magnetic properties of substituted SFO. We found that in $\mathrm{SrFe}_{11.5} \mathrm{Zn}_{0.5} \mathrm{O}_{19}, \mathrm{Zn}$ atoms prefer to occupy $4 f_{1}, 12 k$, and $2 a$ sites with occupation probability of $78 \%, 19 \%$ and $3 \%$, respectively, while in $\mathrm{SrFe}_{11.5} \mathrm{SnO}_{19}$, Sn atoms occupy the $12 k$ and $4 f_{2}$ sites with occupation probability of $54 \%$ and $46 \%$, respectively. We further showed that in $\mathrm{SrFe}_{11} \mathrm{Zn}_{0.5} \mathrm{Sn}_{0.5} \mathrm{O}_{19}$, the pair of $(\mathrm{Zn}, \mathrm{Sn})$ atoms prefers to occupy the $\left(4 f_{1}, 4 f_{2}\right),\left(4 f_{1}, 12 k\right)$ and $(12 k, 12 k)$ sites with occupation probability of $82 \%, 8 \%$ and $6 \%$, respectively. The results from our model based on the formation probability were found to be in good agreement with recent experimental observations showing enhancement of magnetization and the reduction in anisotropy for Zn-Sn substituted strontium hexaferrites.

\section{ACKNOWLEDGMENT}

This research was supported by the Creative Materials Discovery Program through the National Research Foundation of Korea (NRF) funded by the Ministry of Science, ICT, and Future Planning (2016M3D1A1919181). It was also partly supported by the Center for Computational Sciences (CCS) at Mississippi State University. YKH's work was partially supported by National Science Foundation (NSF) under Award Number CMMI 1463301. Computer time allocation has been provided by the High Performance Computing Collaboratory $\left(\mathrm{HPC}^{2}\right)$ at Mississippi State University. 
[1] Zhiyong Pang, Xijian Zhang, Boming Ding, Daxin Bao, and Baoshan Han, "Microstructure and magnetic microstructure of La+Co doped strontium hexaferrites," Journal of Alloys and Compounds 492, 691-694 (2010).

[2] A. Davoodi and B. Hashemi, "Magnetic properties of $\mathrm{SnMg}$ substituted strontium hexaferrite nanoparticles synthesized via coprecipitation method," Journal of Alloys and Compounds 509, 5893-5896 (2011).

[3] Muhammad Naeem Ashiq, Muhammad Javed Iqbal, Muhammad Najam ul Haq, Pablo Hernandez Gomez, and Ashfaq Mahmood Qureshi, "Synthesis, magnetic and dielectric properties of ErNi doped Sr-hexaferrite nanomaterials for applications in High density recording media and microwave devices," Journal of Magnetism and Magnetic Materials 324, 15 - 19 (2012).

[4] Suriya Ounnunkad, "Improving magnetic properties of barium hexaferrites by La or Pr substitution," Solid State Communications 138, $472-475$ (2006)

[5] J.F. Wang, C.B. Ponton, and I.R. Harris, "A study of Prsubstituted strontium hexaferrite by hydrothermal synthesis," Journal of Alloys and Compounds 403, 104-109 (2005)

[6] J.F Wang, C.B Ponton, R Grössinger, and I.R Harris, "A study of La-substituted strontium hexaferrite by hydrothermal synthesis," Journal of Alloys and Compounds 369, 170-177 (2004)

[7] D. Seifert, J. Tpfer, F. Langenhorst, J.-M. Le Breton, H. Chiron, and L. Lechevallier, "Synthesis and magnetic properties of La-substituted M-type Sr hexaferrites," Journal of Magnetism and Magnetic Materials 321, 4045 - 4051 (2009).

[8] J.F. Wang, C.B. Ponton, and I.R. Harris, "A study of the magnetic properties of hydrothermally synthesised $\mathrm{Sr}$ hexaferrite with Sm substitution," Journal of Magnetism and Magnetic Materials 234, 233 - 240 (2001)

[9] J.F. Wang, C.B. Ponton, and I.R. Harris, "A study of Nd-substituted Sr hexaferrite prepared by hydrothermal synthesis," IEEE Transactions on Magnetics 38, 29282930 (2002)

[10] Vivek Dixit, Chandani N. Nandadasa, Seong-Gon Kim, Sungho Kim, Jihoon Park, Yang-Ki Hong, Laalitha S. I. Liyanage, and Amitava Moitra, "Site occupancy and magnetic properties of Al-substituted M-type strontium hexaferrite," Journal of Applied Physics 117, 243904 (2015)

[11] G. Albanese, M. Carbucicchio, and A. Deriu, "Temperature dependence of the sublattice magnetizations in Aland Ga-substituted M-type hexagonal ferrites," Physica Status Solidi (a) 23, 351-358 (1974)

[12] M. Awawdeh, I. Bsoul, and S.H. Mahmood, "Magnetic properties and Mssbauer spectroscopy on $\mathrm{Ga}, \mathrm{Al}$, and Cr substituted hexaferrites," Journal of Alloys and Compounds 585, 465 - $473(2014)$

[13] H.Z. Wang, B. Yao, Y. Xu, Q. He, G.H. Wen, S.W. Long, J. Fan, G.D. Li, L. Shan, B. Liu, L.N. Jiang, and L.L. Gao, "Improvement of the coercivity of strontium hexaferrite induced by substitution of $\mathrm{Al}^{3+}$ ions for $\mathrm{Fe}^{3+}$ ions," Journal of Alloys and Compounds 537, 43 - 49 (2012)

[14] Lev A. Trusov, Evgeny A. Gorbachev, Vasily A. Lebedev, Anastasia E. Sleptsova, Ilya V. Roslyakov, Ekate- rina S. Kozlyakova, Alexander V. Vasiliev, Robert E. Dinnebier, Martin Jansen, and Pavel E. Kazin, "CaAl double-substituted strontium hexaferrites with giant coercivity," Chem. Commun. 54, 479-482 (2018).

[15] Qingqing Fang, "Temperature dependence of magnetic properties of zinc and niobium doped strontium hexaferrite nanoparticles," Journal of Applied Physics 95, 6360 (2004).

[16] H.C. Fang, Z. Yang, C.K. Ong, Y. Li, and C.S. Wang, "Preparation and magnetic properties of ( $\mathrm{ZnSn})$ substituted barium hexaferrite nanoparticles for magnetic recording," Journal of Magnetism and Magnetic Materials 187, $129-135(1998)$

[17] G Mendoza-Surez, K.K Johal, H Mancha-Molinar, J.I Escalante-Garca, and M.M Cisneros-Guerrero, "Magnetic properties of $\mathrm{Zn}$-Sn-substituted Ba-ferrite powders prepared by ball milling," Materials Research Bulletin 36, 2597 - 2603 (2001)

[18] Ali Ghasemi, Vladimir epelk, Xiaoxi Liu, and Akimitsu Morisako, "The role of cations distribution on magnetic and reflection loss properties of ferrimagnetic $\mathrm{SrFe}_{12 x}\left(\mathrm{Sn}_{0.5} \mathrm{Zn}_{0.5}\right)_{x} \mathrm{O}_{19}$," Journal of Applied Physics 107, 09A734 (2010), https://doi.org/10.1063/1.3338988.

[19] Ali Ghasemi and Vladimir Sepelák, "Correlation between site preference and magnetic properties of substituted strontium ferrite thin films," Journal of Magnetism and Magnetic Materials 323, 1727-1733 (2011).

[20] C.M. Fang, F. Kools, R. Metselaar, R.A. Groot, and Others, "Magnetic and electronic properties of strontium hexaferrite $\mathrm{SrFe}_{12} \mathrm{O}_{19}$ from first-principles calculations," Journal of Physics: Condensed Matter 15, 6229-6237 (2003)

[21] Jihoon Park, Yang-Ki Hong, Seong-Gon Kim, Sungho Kim, Laalitha S.I. Liyanage, Jaejin Lee, Woncheol Lee, Gavin S. Abo, Kang-Heon Hur, and Sung-Yong An, "Maximum energy product at elevated temperatures for hexagonal strontium ferrite $\left(\mathrm{SrFe}_{12} \mathrm{O}_{19}\right)$ magnet," Journal of Magnetism and Magnetic Materials 355, $1-6$ (2014)

[22] M. Küpferling, P. Novák, K. Knížek, M. W. Pieper, R. Grössinger, G. Wiesinger, and M. Reissner, "Magnetism in La substituted Sr hexaferrite," Journal of Applied Physics 97, 10F309 (2005).

[23] P. Novák, K. Knížek, M. Küpferling, R. Grössinger, and M. W. Pieper, "Magnetism of mixed valence (LaSr) hexaferrites," The European Physical Journal B - Condensed Matter and Complex Systems 43, 509-515 (2005), 10.1140/epjb/e2005-00084-8.

[24] Laalitha S.I. Liyanage, Sungho Kim, Yang-Ki Hong, JiHoon Park, Steven C. Erwin, and Seong-Gon Kim, "Theory of magnetic enhancement in strontium hexaferrite through ZnSn pair substitution," Journal of Magnetism and Magnetic Materials 348, 75 - 81 (2013)

[25] Vivek Dixit, Chandani N. Nandadasa, Seong-Gon Kim, Sungho Kim, Jihoon Park, and Yang-Ki Hong, "Site preference and magnetic properties of $\mathrm{Ga} / \mathrm{In}$-substituted strontium hexaferrite: An ab initio study," Journal of Applied Physics 118, 203908 (2015)

[26] Ali Ghasemi, Vladimir Sepelák, Xiaoxi Liu, and Akimitsu Morisako, "The role of cations distribution on magnetic and reflection loss properties of ferrimagnetic 
$\mathrm{SrFe}_{12 x}\left(\mathrm{Sn}_{0.5} \mathrm{Zn}_{0.5}\right)_{x} \mathrm{O}_{19}$," Journal of Applied Physics 107, 09A734 (2010)

[27] X. Obradors, X. Solans, A. Collomb, D. Samaras, J. Rodriguez, M. Pernet, and M. Font-Altaba, "Crystal structure of strontium hexaferrite $\mathrm{SrFe}_{12} \mathrm{O}_{19}$," Journal of Solid State Chemistry 72, $218-224$ (1988)

[28] G. Kresse and J. Furthmüller, "Efficient iterative schemes for $a b$ initio total-energy calculations using a plane-wave basis set," Phys. Rev. B 54, 11169-11186 (1996)

[29] G. Kresse and D. Joubert, "From ultrasoft pseudopotentials to the projector augmented-wave method," Phys. Rev. B 59, 1758-1775 (1999)

[30] John P. Perdew, Kieron Burke, and Matthias Ernzerhof, "Generalized Gradient Approximation Made Simple," Phys. Rev. Lett. 77, 3865-3868 (1996).

[31] E. F. Gorter, "Saturation magnetization of some ferrimagnetic oxides with hexagonal crystal structures," Proc. IEEE 104B, 2555 (1957).

[32] H.J. Monkhorst and J.D. Pack, "Special points for Brillouin-zone integrations," Phys. Rev. B 13, 5188-5192 (1976)

[33] M. Methfessel and A. T. Paxton, "High-precision sampling for Brillouin-zone integration in metals," Phys. Rev. B 40, 3616-3621 (1989).

[34] Peter E. Blöchl, O. Jepsen, and O. K. Andersen, "Improved tetrahedron method for Brillouin-zone integrations," Phys. Rev. B 49, 16223-16233 (1994)

[35] S. L. Dudarev, G. A. Botton, S. Y. Savrasov, C. J. Humphreys, and A. P. Sutton, "Electron-energy-loss spectra and the structural stability of nickel oxide: An LSDA+U study," Phys. Rev. B 57, 1505-1509 (1998).

[36] Koichi Momma and Fujio Izumi, "VESTA3 for threedimensional visualization of crystal, volumetric and morphology data," Journal of Applied Crystallography 44, 1272-1276 (2011)

[37] P. Ravindran, A. Delin, P. James, B. Johansson, J. M. Wills, R. Ahuja, and O. Eriksson, "Magnetic, optical, and magneto-optical properties of $\mathrm{MnX}(\mathrm{X}=\mathrm{As}, \mathrm{Sb}$, or Bi) from full-potential calculations," Phys. Rev. B 59, 15680-15693 (1999)
[38] G. H. O. Daalderop, P. J. Kelly, and M. F. H. Schuurmans, "First-principles calculation of the magnetocrystalline anisotropy energy of iron, cobalt, and nickel," Phys. Rev. B 41, 11919-11937 (1990)

[39] F. Muñoz, A.H. Romero, J. Mejía-López, and J.L. Morán-López, "Finite size effects on the magnetocrystalline anisotropy energy in Fe magnetic nanowires from first principles," Journal of Nanoparticle Research 15, 1524 (2013), 10.1007/s11051-013-1524-6.

[40] J. Smit and H.P.J. Wijn, Ferrites (Philips Technical Library, Eindhoven, 1959).

[41] Charles Kittel, "Physical Theory of Ferromagnetic Domains," Rev. Mod. Phys. 21, 541-583 (1949).

[42] Vivek Dixit, Seong-Gon Kim, Jihoon Park, and Yang-Ki Hong, "Effect of ionic substitutions on the magnetic properties of strontium hexaferrite: A first principles study," AIP Advances 7, 115209 (2017), https://doi.org/10.1063/1.4995309.

[43] Charles M. Gilmore, Materials science and engineering properties (Cengage Learning, 2014).

[44] Z. W. Li, C. K. Ong, Z. Yang, F. L. Wei, X. Z. Zhou, J. H. Zhao, and A. H. Morrish, "Site preference and magnetic properties for a perpendicular recording material: $\mathrm{BaFe}_{12-x} \mathrm{Zn}_{x / 2} \mathrm{Zr}_{x / 2} \mathrm{O}_{19}$ nanoparticles," Phys. Rev. B 62, 6530-6537 (2000)

[45] X. Obradors, A. Collomb, M. Pernet, D. Samaras, and J.C. Joubert, "X-ray analysis of the structural and dynamic properties of $\mathrm{BaFe}_{12} \mathrm{O}_{19}$ hexagonal ferrite at room temperature," Journal of Solid State Chemistry 56, 171 -181 (1985)

[46] D.A. Vinnik, A.S. Semisalova, L.S. Mashkovtseva, A.K. Yakushechkina, S. Nemrava, S.A. Gudkova, D.A. Zherebtsov, N.S. Perov, L.I. Isaenko, and R. Niewa, "Growth, structural and magnetic characterization of Znsubstituted barium hexaferrite single crystals," Materials Chemistry and Physics 163, 416 - 420 (2015).

[47] A Gonzlez-Angeles, G Mendoza-Surez, A Gruskov, R Dosoudil, and R Ortega-Zempoalteca, "Magnetic studies of $\mathrm{Sn}^{2+} \mathrm{Sn}^{4+}$-substituted barium hexaferrites synthesized by mechanical alloying," Materials Letters 58, $2906-2910(2004)$ 